\title{
Entropy solution for a nonlinear parabolic problem with homogeneous Neumann boundary condition involving variable exponents
}

\author{
U. TRAORÉ ${ }^{1}$ \\ 1 Laboratoire de Mathématiques et \\ Informatique (LAMI), Université Joseph \\ KI-ZERBO 03, BP 7021 Ouaga 03, \\ Ouagadougou, Burkina Faso. \\ urbain.traore@yahoo.fr
}

\begin{abstract}
In this paper we prove the existence and uniqueness of an entropy solution for a non-linear parabolic equation with homogeneous Neumann boundary condition and initial data in $L^{1}$. By a time discretization technique we analyze the existence, uniqueness and stability questions. The functional setting involves Lebesgue and Sobolev spaces with variable exponents.
\end{abstract}

\section{RESUMEN}

En este artículo probamos la existencia y unicidad de una solución de entropía para una ecuación parabólica no lineal con condiciones de borde Neumann homogéneas y data inicial en $L^{1}$. Usando una técnica de discretización del tiempo, analizamos las preguntas de existencia, unicidad y estabilidad. El contexto funcional involucra espacios de Lebesgue y Sobolev con exponentes variables.

Keywords and Phrases: Nonlinear parabolic problem, variable exponents, entropy solution, Neumann-type boundary conditions, semi-discretization.

2020 AMS Mathematics Subject Classification: 35K55, 35K61, 35J60, 35Dxx. 


\section{Introduction and main result}

Let $\Omega$ be a smooth bounded open domain of $\mathbb{R}^{d},(d \geq 3)$ with Lipschitz boundary $\partial \Omega, T$ is a fixed positive number, in this paper we study the existence and uniqueness of an entropy solution for the following nonlinear parabolic problem

$$
(P)\left\{\begin{array}{l}
\left.\frac{\partial u}{\partial t}-\operatorname{div} a(x, \nabla u)+b(u)=f \text { in } Q_{T}=\right] 0, T[\times \Omega, \\
\left.a(x, \nabla u) \cdot \eta=0 \text { on } \sum_{T}=\right] 0, T[\times \partial \Omega, \\
u(0, .)=u_{0} \text { in } \Omega,
\end{array}\right.
$$

where $f \in L^{1}\left(Q_{T}\right), b: \mathbb{R} \rightarrow \mathbb{R}, a(x, \xi): \Omega \times \mathbb{R}^{d} \rightarrow \mathbb{R}$ is Carathéodory function and verifying some assumptions which will be given later, $\eta$ denotes the unit vector normal on $\partial \Omega$.

The usual weak formulations of parabolic problems in the case where the initial data are in $L^{1}$ do not ensure existence and uniqueness of solutions. For this reason, new formulations and types of solutions are given in order to obtain existence and uniqueness. For that, three notions of solution have been adopted: solutions named SOLA (Solution Obtained as the Limit of Approximations) defined by A. Dall'Aglio (see [10]); renormalized solutions defined by R. DiPerna and P.-L. Lions (see [12]); and entropy solutions defined by Ph. Bénilan et al. in [8]. In this paper, we will be interested in the entropy formulation.

The stationary version of the problem for the problem $(P)$ has been already studied by Bonzi et al. (cf. [9]), where they proved the existence and uniqueness of an entropy solution for the initial data in $L^{1}$.

The study of parabolic equations with variable exponents is a very active field (see $[1,2,20,21$, $23,27,29]$ ), in these papers, the authors consider the homogeneous Dirichlet boundary conditions, which permit them to use many results in the generalized Sobolev space $W^{1, p(.)}(\Omega)$ and the many results concerned the differential equation in the literature to achieve there works. In particular in the case of $p(x)$-Laplace, where $b \equiv 0$, Bendahmane et al. (see [6]) have proved the existence and uniqueness of renormalized solution. We can also point out that the well-posedness of triply nonlinear degenerate elliptic- parabolic-hyperbolic problems: $b(u)_{t}-\operatorname{div} a(x, \nabla \phi(u))+\psi(u)=f$ in a bounded domain with homogeneous Dirichlet boundary conditions by K. H. Karlsen et al. in [3]. Unfortunately, in this paper, due to the Neumann boundary condition, we cannot use the ideas developed in these papers and also some functional analysis results which play and important role in the a priori estimation, in particular the famous Poincaré inequality.

To overcome these difficulties we apply a time discretization of given continuous problem by the Euler forward scheme. Let's recall that this method has been used in the literature for the study 
of some nonlinear parabolic problems, we refer for example to $[7,13,16,17]$ for some details. This scheme is usually used to prove existence of solutions as well as to compute numerical approximations.

In this paper, our assumptions are the following:

$$
\left\{\begin{aligned}
p(.): \bar{\Omega} & \rightarrow \mathbb{R} \text { is a continuous function such that } \\
1<p_{-} & \leq p_{+}<+\infty,
\end{aligned}\right.
$$

where $p_{-}:=e s s \inf _{x \in \Omega} p(x)$ and $p_{+}:=e s s \sup _{x \in \Omega} p(x)$ and

$b: \bar{\Omega} \rightarrow \mathbb{R}$ is a continuous, nondecreasing function, surjective such that $b(0)=0$.

Also, we assume that $a(x, \xi): \Omega \times \mathbb{R}^{N} \rightarrow \mathbb{R}^{N}$ is Carathéodory such that:

- there exists a positive constant $C_{1}$ with

$$
|a(x, \xi)| \leq C_{1}\left(j(x)+|\xi|^{p(x)-1}\right)
$$

for almost every $x \in \Omega$ and for every $\xi \in \mathbb{R}^{N}$, where $j$ is a nonnegative function in $L^{p^{\prime}(.)}(\Omega)$ with $\frac{1}{p(x)}+\frac{1}{p^{\prime}(x)}=1$

- there exists a positive constant $C_{2}$ such that for every $x \in \Omega$ and every $\xi_{1}, \xi_{2} \in \mathbb{R}^{d}$ with $\xi_{1} \neq \xi_{2}$, the following two inequalities hold

$$
\begin{gathered}
\left(a\left(x, \xi_{1}\right)-a\left(x, \xi_{2}\right)\right) \cdot\left(\xi_{1}-\xi_{2}\right)>0 \\
a(x, \xi) \cdot \xi \geq C_{2}|\xi|^{p(x)} .
\end{gathered}
$$

The rest of the paper is organized as follows: after some preliminary results in Section 2, we introduce the Euler forward scheme associated with the problem $(P)$ in Section 3. We analyze the stability of the discretized problem and we study the existence of an entropy solution to the parabolic problem $(P)$ in the Section 4.

\section{Preliminaries}

We define the Lebesgue space with variable exponent $L^{p(.)}(\Omega)$ (see [11]) as the set of all measurable functions $u: \Omega \rightarrow \mathbb{R}$ for which the convex modular

$$
\rho_{p(.)}(u):=\int_{\Omega}|u|^{p(x)} d x
$$

is finite.

If the exponent is bounded, i.e., if $p_{+}<+\infty$, then the expression

$$
\|u\|_{p(.)}:=\inf \left\{\lambda>0: \rho_{p(.)}(u / \lambda) \leq 1\right\}
$$


defines a norm in $L^{p(.)}(\Omega)$, called the Luxembourg norm.

The space $\left(L^{p(.)}(\Omega),\|\cdot\|_{p(.)}\right)$ is a separable Banach space. Moreover, if $1<p_{-} \leq p_{+}<+\infty$, then $L^{p(.)}(\Omega)$ is uniformly convex, hence reflexive and its dual space is isomorphic to $L^{p^{\prime}(.)}(\Omega)$, where $\frac{1}{p(x)}+\frac{1}{p^{\prime}(x)}=1$.

Finally, we have the Hölder type inequality

$$
\left|\int_{\Omega} u v d x\right| \leq\left(\frac{1}{p_{-}}+\frac{1}{\left(p_{-}\right)^{\prime}}\right)\|u\|_{p(.)}\|v\|_{p^{\prime}(.)},
$$

for all $u \in L^{p(.)}(\Omega)$ and $v \in L^{p^{\prime}(.)}(\Omega)$.

Let

$$
W^{1, p(.)}(\Omega):=\left\{u \in L^{p(.)}(\Omega):|\nabla u| \in L^{p(.)}(\Omega)\right\},
$$

which is Banach space equipped with the following norm

$$
\|u\|_{1, p(.)}:=\|u\|_{p(.)}+\|\nabla u\|_{p(.)} .
$$

The space $\left(W^{1, p(.)}(\Omega),\|\cdot\|_{1, p(.)}\right)$ is a separable and reflexive Banach space.

An important role in manipulating the generalized Lebesgue and Sobolev spaces is played by the modular $\rho_{p(.)}$ of the space $L^{p(.)}(\Omega)$. We have the following result.

Proposition 2.1 (see $[14,28]$ ). If $u_{n}, u \in L^{p(.)}(\Omega)$ and $p_{+}<\infty$, the following properties hold true:

(i) $\|u\|_{p(.)}>1 \Rightarrow\|u\|_{p(.)}^{p_{-}}<\rho_{p(.)}(u)<\|u\|_{p(.)}^{p_{+}} ;$

(ii) $\|u\|_{p(.)}<1 \Rightarrow\|u\|_{p(.)}^{p_{+}}<\rho_{p(.)}(u)<\|u\|_{p(.)}^{p_{-}}$;

(iii) $\|u\|_{p(.)}<1$ (respectively $\left.=1 ;>1\right) \Leftrightarrow \rho_{p(.)}(u)<1$ (respectively $\left.=1 ;>1\right)$;

(iv) $\left\|u_{n}\right\|_{p(.)} \rightarrow 0$ (respectively $\left.\rightarrow+\infty\right) \Leftrightarrow \rho_{p(.)}\left(u_{n}\right)<1$ (respectively $\left.\rightarrow+\infty\right)$;

(v) $\rho_{p(.)}\left(u /\|u\|_{p(.)}\right)=1$.

For a measurable function $u: \Omega \rightarrow \mathbb{R}$ we introduce the following notation:

$$
\rho_{1, p(.)}(u)=\int_{\Omega}|u|^{p(x)} d x+\int_{\Omega}|\nabla u|^{p(x)} d x .
$$

Proposition 2.2 (see $[25,26]$ ). If $u \in W^{1, p(.)}(\Omega)$, the following properties hold true:

(i) $\|u\|_{1, p(.)}>1 \Rightarrow\|u\|_{1, p(.)}^{p_{-}}<\rho_{1, p(.)}(u)<\|u\|_{1, p(.)}^{p_{+}}$;

(ii) $\|u\|_{1, p(.)}<1 \Rightarrow\|u\|_{1, p(.)}^{p_{+}}<\rho_{p(.)}(u)<\|u\|_{1, p(.)}^{p_{-}}$;

(iii) $\|u\|_{1, p(.)}<1$ (respectively $\left.=1 ;>1\right) \Leftrightarrow \rho_{1, p(.)}(u)<1($ respectively $=1 ;>1)$. 
Put

$$
p^{\partial}(x):=(p(x))^{\partial}=\left\{\begin{array}{rll}
\frac{(N-1) p(x)}{N-p(x)}, & \text { if } & p(x)<N \\
\infty, & \text { if } & p(x) \geq N .
\end{array}\right.
$$

Proposition 2.3 (see $[26])$. Let $p \in C(\bar{\Omega})$ and $p_{-}>1$. If $q \in C(\partial \Omega)$ satisfies the condition

$$
1<q(x)<p^{\partial}(x) \quad \forall x \in \partial \Omega
$$

then, there is a compact embedding $W^{1, p(.)}(\Omega) \hookrightarrow L^{q(.)}(\partial \Omega)$.

In particular, there is a compact embedding $W^{1, p(.)}(\Omega) \hookrightarrow L^{p(.)}(\partial \Omega)$.

Following [29], we extend a variable exponent $p: \bar{\Omega} \rightarrow[1,+\infty)$ to $\overline{Q_{T}}=[0, T] \times \bar{\Omega}$ by setting $p(t, x)=p(x)$ for all $(t, x) \in \overline{Q_{T}}$.

We may also consider the generalized Lebesgue space

$$
L^{p(.)}(Q)=\left\{u: Q \rightarrow \mathbb{R} \text { measurable such that } \iint_{Q}|u(t, x)|^{p(x)} d(t, x)<\infty\right\}
$$

endowed with the norm

$$
\|u\|_{L^{p(.)}\left(Q_{T}\right)}:=\inf \left\{\lambda>0, \iint_{Q_{T}}\left|\frac{u(t, x)}{\lambda}\right|^{p(x)} d(t, x)<1\right\},
$$

which share the same properties as $L^{p(\cdot)}(\Omega)$.

For a measurable set $U$ in $\mathbb{R}^{d}$, meas $(U)$ denotes its measure, $C_{i}$ and $C$ will denote various positive constants. For a Banach space $X$ and $a<b, L^{q}(a, b ; X)$ is the space of measurable functions $u:[a, b] \rightarrow X$ such that

$$
\left(\int_{a}^{b}\|u\|_{X}^{q} d t\right)^{\frac{1}{q}}:=\|u\|_{L^{q}(a, b ; X)}<\infty
$$

For a given constant $k>0$ we define the cut-off function $T_{k}: \mathbb{R} \rightarrow \mathbb{R}$ by

$$
T_{k}(s):=\left\{\begin{array}{rll}
s & \text { if } & |s| \leq k \\
k \operatorname{sign}(s) & \text { if } & |s|>k
\end{array}\right.
$$

with

$$
\operatorname{sign}(s):=\left\{\begin{array}{rcc}
1 & \text { if } & s>0 \\
0 & \text { if } & s=0 \\
-1 & \text { if } & s<0
\end{array}\right.
$$

Let $J_{k}: \mathbb{R} \rightarrow \mathbb{R}^{+}$defined by

$$
J_{k}(x)=\int_{0}^{x} T_{k}(s) d s
$$

( $J_{k}$ is a primitive of $T_{k}$ ). We have (see [15])

$$
\left\langle\frac{\partial v}{\partial t}, T_{k}(s)\right\rangle=\frac{d}{d t}\left(\int_{\Omega} J_{k}(v) d x\right) \text { in } L^{1}(] 0, T[)
$$


which implies that

$$
\int_{0}^{t}\left\langle\frac{\partial v}{\partial t}, T_{k}(s)\right\rangle=\int_{\Omega} J(v(t)) d x-\int_{\Omega} J(v(0)) d x
$$

For all $u \in W^{1, p(.)}(\Omega)$ we denote by $\tau(u)$ the trace of $u$ on $\partial \Omega$ in the usual sense.

In the sequel, we will identify at the boundary, $u$ and $\tau(u)$.

Set

$$
\mathcal{T}^{1, p(.)}(\Omega)=\left\{u: \Omega \rightarrow \mathbb{R}, \text { measurable such that } T_{k}(u) \in W^{1, p(.)}(\Omega) \text {, for any } k>0\right\} .
$$

Proposition 2.4 (see $[8]$ ). Let $u \in \mathcal{T}^{1, p(.)}(\Omega)$. Then there exists a unique measurable function $v: \Omega \rightarrow \mathbb{R}^{N}$ such that $\nabla T_{k}(u)=v \chi_{\{|u|<k\}}$, for all $k>0$. The function $v$ is denoted by $\nabla u$. Moreover, if $u \in W^{1, p(.)}(\Omega)$ then $v \in\left(L^{p(.)}(\Omega)\right)^{N}$ and $v=\nabla u$ in the usual sense.

We denote by $\mathcal{T}_{t r}^{1, p(.)}(\Omega)(c f . \quad[4,5,18,19])$ the set of functions $u \in \mathcal{T}^{1, p(.)}(\Omega)$ such that there exists a sequence $\left(u_{n}\right)_{n \in \mathbb{N}} \subset W^{1, p(.)}(\Omega)$ satisfying the following conditions:

i) $u_{n} \rightarrow u$ a.e. in $\Omega$.

ii) $\nabla T_{k}\left(u_{n}\right) \rightarrow \nabla T_{k}(u)$ in $\left(L^{1}(\Omega)\right)^{N}$ for any $k>0$.

iii) There exists a measurable function $v$ on $\partial \Omega$, such that $u_{n} \rightarrow v$ a.e. on $\partial \Omega$.

The function $v$ is the trace of $u$ in the generalized sense introduced in $[4,5]$. In the sequel, the trace of $u \in \mathcal{T}_{t r}^{1, p(.)}(\Omega)$ on $\partial \Omega$ will be denoted by $\operatorname{tr}(u)$. If $u \in W^{1, p(.)}(\Omega), \operatorname{tr}(u)$ coincides with $\tau(u)$ in the usual sense. Moreover $u \in \mathcal{T}_{t r}^{1, p(.)}(\Omega)$ and for every $k>0, \tau\left(T_{k}(u)\right)=T_{k}(\operatorname{tr}(u))$ and if $\varphi \in W^{1, p(.)}(\Omega) \cap L^{\infty}(\Omega)$ then $(u-\varphi) \in \mathcal{T}_{t r}^{1, p(.)}(\Omega)$ and $\operatorname{tr}(u-\varphi)=\operatorname{tr}(u)-\operatorname{tr}(\varphi)$.

\section{The semi-discrete problem}

In this section, we study the Euler forward scheme associated with the problem $(P)$ :

$$
\left(P_{n}\right)\left\{\begin{array}{l}
U^{n}-\tau \operatorname{div} a\left(x, \nabla U^{n}\right)+\tau b\left(U^{n}\right)=\tau f^{n}+U^{n-1} \text { in } \Omega \\
a\left(x, \nabla U^{n}\right) \cdot \eta=0 \text { on } \partial \Omega \\
U^{0}=u_{0} \text { in } \Omega
\end{array}\right.
$$

where $N \tau=T, 0<\tau<1,1 \leq n \leq N$ and

$$
f_{n}(.)=\frac{1}{\tau} \int_{(n-1) \tau}^{n \tau} f(s, .) d s \text { in } \Omega .
$$


Definition 3.1. An entropy solution to the discretized problems $\left(P_{n}\right)$ is a sequence $\left(U^{n}\right)_{0 \leq n \leq N}$ such that $U^{0}=u_{0} \in L^{1}(\Omega)$ and $U^{n}$ is defined by induction as an entropy solution to the problem

$$
\left\{\begin{array}{l}
U^{n}-\tau \operatorname{div} a\left(x, \nabla U^{n}\right)+\tau b\left(U^{n}\right)=\tau f_{n}+U^{n-1} \text { in } \Omega \\
a\left(x, \nabla U^{n}\right) \cdot \eta=0 \text { on } \partial \Omega
\end{array}\right.
$$

i.e. $U^{n} \in \mathcal{T}_{\text {tr }}^{1, p(.)}(\Omega), b\left(U^{n}\right) \in L^{1}(\Omega)$, and for every $k>0$

$\tau \int_{\Omega} a\left(x, \nabla U^{n}\right) \cdot \nabla T_{k}\left(U^{n}-\varphi\right) d x+\int_{\Omega}\left(\tau b\left(U^{n}\right)+U^{n}\right) T_{k}\left(U^{n}-\varphi\right) d x \leq \int_{\Omega}\left(\tau f_{n}+U^{n-1}\right) T_{k}\left(U^{n}-\varphi\right) d x$

for all $\varphi \in W^{1, p(.)}(\Omega) \cap L^{\infty}(\Omega)$.

We have the following result

Lemma 3.2. Let hypotheses $(1.3)-(1.5)$ be satisfied. If $\left(U^{n}\right)_{0 \leq n \leq N}$ is an entropy solution of problems $\left(P_{n}\right)$, then $U^{n} \in L^{1}(\Omega)$ for all $n=1, \ldots, N$.

Proof. For $n=1$, we take $\varphi=0$ in (3.1), to get,

$$
\tau \int_{\Omega} a\left(x, \nabla U^{1}\right) \cdot \nabla T_{k}\left(U^{1}\right) d x+\int_{\Omega}\left(\tau b\left(U^{1}\right)+U^{1}\right) T_{k}\left(U^{1}\right) d x \leq \int_{\Omega}\left(\tau f_{1}+u_{0}\right) T_{k}\left(U^{1}\right) d x,
$$

which is equivalent to

$\tau \int_{\Omega} a\left(x, \nabla T_{k}\left(U^{1}\right)\right) \nabla T_{k}\left(U^{1}\right) d x+\int_{\Omega} \tau b\left(U^{1}\right) T_{k}\left(U^{1}\right) d x+\int_{\Omega} U^{1} T_{k}\left(U^{1}\right) d x \leq \int_{\Omega}\left(\tau f_{1}+u_{0}\right) T_{k}\left(U^{1}\right) d x$,

By the assumption (1.5) and the properties of the function $b$, we have

$$
\tau \int_{\Omega} a\left(x, \nabla T_{k}\left(U^{1}\right)\right) \nabla T_{k}\left(U^{1}\right) d x+\int_{\Omega} \tau b\left(U^{1}\right) T_{k}\left(U^{1}\right) d x \geq 0,
$$

then it follows that

$$
\int_{\Omega} U^{1} T_{k}\left(U^{1}\right) d x \leq k \tau\left\|f_{1}\right\|_{1}+k\left\|u_{0}\right\|_{1}
$$

Since

$$
\sum_{n=1}^{N} \tau\left\|f_{n}\right\|_{1} \leq\|f\|_{1} .
$$

Then, it follows that

$$
\int_{\Omega} U^{1} T_{k}\left(U^{1}\right) d x \leq k\left(\|f\|_{1}+\left\|u_{0}\right\|_{1}\right)
$$

Since

$$
\lim _{k \rightarrow 0} U^{1} \frac{T_{k}\left(U^{1}\right)}{k}=\left|U^{1}\right| .
$$

Then dividing (3.3) by $k$ and letting $k \rightarrow 0$; we deduce by Fatou's lemma that

$$
\left\|U^{1}\right\|_{1} \leq\left(\|f\|_{1}+\left\|u_{0}\right\|_{1}\right)
$$


Theorem 3.3. Let hypotheses (1.3) - (1.5) be satisfied. Then for all $N \in \mathbb{N}$, the problems $\left(P_{n}\right)$ have unique entropy solution $U^{n} \in \mathcal{T}_{\text {tr }}^{1, p(.)}(\Omega) \cap L^{1}(\Omega)$ for all $n=1, \ldots, N$.

Proof. The problem $\left(P_{1}\right)$ can be rewritten in the following form

$$
\begin{aligned}
& -\tau \operatorname{div} a(x, \nabla u)+\bar{b}(u)=F_{1} \text { in } \Omega \\
& a(x, \nabla u) . \eta=0 \text { on } \partial \Omega
\end{aligned}
$$

with

$$
\bar{b}(s):=\tau b(s)+s, \quad F_{1}:=\tau f_{1}+u_{0} .
$$

From the assumption $\left(H_{2}\right)$, we have $F_{1} \in L^{1}(\Omega)$, and using the properties of $b$, we obtain $\bar{b}$ is a continuous, nondecreasing function, surjective such that $b(0)=0$. Hence, using [9, Theorem 4.3], we have the existence of unique entropy solution $U^{1} \in \mathcal{T}_{t r}^{1, p(.)}(\Omega), b\left(U^{1}\right) \in L^{1}(\Omega)$.

Thanks to Lemma 3.2, by induction, we deduce that for $n=2, \ldots, N$, the problem

$$
\begin{aligned}
& u-\tau \operatorname{div} a(x, \nabla u)+\tau \alpha(u)=\tau f_{n}+U^{n-1} \text { in } \Omega \\
& a(x, \nabla u) \cdot \eta=0 \text { on } \partial \Omega,
\end{aligned}
$$

has an unique entropy solution $U^{n} \in \mathcal{T}_{t r}^{1, p(.)}(\Omega) \cap L^{1}(\Omega), b\left(U^{n}\right) \in L^{1}(\Omega)$.

\section{Stability}

This section is devoted to the a priori estimates for the discrete entropy solution $\left(U^{n}\right)_{1 \leq n \leq N}$. These result are essentials for the study of the convergence of the Euler forward scheme.

Theorem 4.1. Let hypotheses (1.3)-(1.5) be satisfied. Then there exist positive constants $C\left(u_{0}, f\right)$, $C\left(u_{0}, f, \Omega\right)$ depending on the data but not on $N$ such that for all $n=1, \ldots, N$, we have

1. $\left\|U^{n}\right\|_{1} \leq C\left(u_{0}, f\right)$

2. $\tau \sum_{i=1}^{n}\left\|b\left(U^{i}\right)\right\|_{1} \leq C\left(u_{0}, f\right)$

3. $\sum_{i=1}^{n}\left\|U^{i}-U^{i-1}\right\|_{1} \leq C\left(, u_{0}, f\right)$

4. $\tau \sum_{i=1}^{n} \rho_{p(.)}\left(\nabla T_{k}\left(U^{i}\right)\right) \leq k C\left(u_{0}, f\right)$

5. $\tau \sum_{i=1}^{n} \int_{\left\{\left|U^{i}\right| \leq k\right\}}\left|\nabla U^{i}\right|^{p_{-}} d x \leq k C\left(u_{0}, f, \Omega\right)$

Proof. 1 and 2. For $\varphi=0$ as a test function in (3.1), we have

$$
\begin{aligned}
& \frac{\tau}{k} \int_{\Omega} a\left(x, \nabla T_{k}\left(U^{i}\right)\right) \nabla T_{k}\left(U^{i}\right) d x+\int_{\Omega} U^{i} \frac{T_{k}\left(U^{i}\right)}{k} d x+\int_{\Omega} \tau b\left(U^{i}\right) \frac{T_{k}\left(U^{i}\right)}{k} d x \\
& \leq \tau\left\|f_{i}\right\|_{1}+\left\|U^{i-1}\right\|_{1} d x .
\end{aligned}
$$


Since

$$
\int_{\Omega} a\left(x, \nabla T_{k}\left(U^{i}\right)\right) \nabla T_{k}\left(U^{i}\right) d x \geq 0
$$

Then, it follows that

$$
\int_{\Omega} U^{i} \frac{T_{k}\left(U^{i}\right)}{k} d x+\int_{\Omega} \tau b\left(U^{i}\right) \frac{T_{k}\left(U^{i}\right)}{k} d x \leq \tau\left\|f_{i}\right\|_{1}+\left\|U^{i-1}\right\|_{1} .
$$

Then letting $k \rightarrow 0$ and using Fatou's lemma, we deduce that

$$
\left\|U^{i}\right\|_{1}+\tau\left\|b\left(U^{i}\right)\right\|_{1} \leq \tau\left\|f_{i}\right\|_{1}+\left\|U^{i-1}\right\|_{1} .
$$

Now, we sum (4.1) from $i=1$ to $n$ to obtain

$$
\left\|U^{n}\right\|_{1}+\tau \sum_{i=1}^{n}\left\|b\left(U^{i}\right)\right\|_{1} \leq\|f\|_{1}+\left\|u_{0}\right\|_{1}
$$

which give, the inequalities 1 and 2 .

3. For $k \geq 1$, we take $\varphi=T_{h}\left(U^{i}-\operatorname{sign}\left(U^{i}-U^{i-1}\right)\right),(h>1)$ as a test function in (3.1), then letting $h \rightarrow \infty$, for $k \geq 1$, we obtain,

$$
\tau \lim _{h \rightarrow \infty} \mathcal{I}(k, h)+\left\|U^{i}-U^{i-1}\right\|_{1} \leq \tau\left(\left\|f_{i}\right\|_{1}+\left\|b\left(U^{i}\right)\right\|_{1}\right)
$$

where

$$
\begin{aligned}
\mathcal{I}(k, h) & :=\int_{\Omega} a\left(x, \nabla U^{i}\right) \nabla T_{k}\left(U^{i}-T_{h}\left(U^{i}-\operatorname{sign}\left(U^{i}-U^{i-1}\right)\right)\right) d x \\
& =\int_{\Omega_{k, h} \cap \overline{\Omega(k)}} a\left(x, \nabla U^{i}\right) \nabla U^{i} d x
\end{aligned}
$$

and

$$
\begin{aligned}
& \Omega_{k, h}:=\left\{\left|U^{i}-T_{h}\left(U^{i}-\operatorname{sign}\left(U^{i}-U^{i-1}\right)\right)\right| \leq k\right\} \\
& \overline{\Omega(k)}=\left\{\left|U^{i}-\operatorname{sign}\left(U^{i}-U^{i-1}\right)\right|>h\right\} .
\end{aligned}
$$

Then by the hypothesis (1.3), we have

$$
\lim _{h \rightarrow \infty} \mathcal{I}(k, h) \geq 0
$$

Then, it follows that

$$
\left\|U^{i}-U^{i-1}\right\|_{1} \leq k \tau\left(\left\|f_{i}\right\|_{1}+\left\|b\left(U^{i}\right)\right\|_{1}\right) .
$$

Then, summing (4.3) from $i=1$ to $n$ and by the stability result 2, we obtain the stability result 3 .

4. We take $\varphi=0$ as a test function in 3.1 to get

$$
\tau\left(\int_{\Omega} \mid a\left(x, \nabla T_{k}\left(U^{i}\right)\right) \nabla T_{k}\left(U^{i}\right) d x\right) \leq k \tau\left(\left\|f_{i}\right\|_{1}+\left\|b\left(U^{i}\right)\right\|_{1}\right)+k\left\|U^{i}-U^{i-1}\right\|_{1} .
$$


Therefore, using the assumption (1.5) it follows that

$$
\tau \rho_{p(x)}\left(\nabla T_{k}\left(U^{i}\right)\right) \leq C_{3}\left[k \tau\left(\left\|f_{i}\right\|_{1}+\left\|b\left(U^{i}\right)\right\|_{1}\right)+k\left\|U^{i}-U^{i-1}\right\|_{1}\right] .
$$

Now, summing (4.4) from $i=1$ to $n$ and using the stability results $1,2,3$, we get

$$
\begin{aligned}
\tau \sum_{i=1}^{n} \rho_{p(x)}\left(\nabla T_{k}\left(U^{i}\right)\right) & \leq C_{3} k\left[\|f\|_{1}+\tau \sum_{i=1}^{n}\left\|b\left(U^{i}\right)\right\|_{1}+\sum_{i=1}^{n}\left\|U^{i}-U^{i-1}\right\|_{1}\right] \\
& \leq k C\left(f, u_{0}\right) .
\end{aligned}
$$

5. According to (4.5), we get from the above estimate

$$
\tau \sum_{i=1}^{n} \int_{\left\{\left|U^{i}\right| \leq k\right\}}\left|\nabla U^{i}\right|^{p(x)} d x \leq k C\left(u_{0}, f\right) .
$$

Now, note that

$$
\begin{aligned}
\int_{\left\{\left|U^{i}\right| \leq k\right\}}\left|\nabla U^{i}\right|^{p_{-}} d x & =\int_{\left\{\left|U^{i}\right| \leq k,\left|\nabla U^{i}\right|>\frac{1}{N}\right\}}\left|\nabla U^{i}\right|^{p_{-}} d x+\int_{\left\{\left|U^{i}\right| \leq k\left|\nabla U^{i}\right| \leq \frac{1}{N}\right\}}\left|\nabla U^{i}\right|^{p_{-}} d x \\
& \leq \int_{\left\{\left|U^{i}\right| \leq k,\left|\nabla U^{i}\right|>\frac{1}{N}\right\}}\left|\nabla U^{i}\right|^{p_{-}} d x+\frac{1}{N} \operatorname{meas}(\Omega) \\
& \leq \int_{\left\{\left|U^{i}\right| \leq k\right\}}\left|\nabla U^{i}\right|^{p(x)} d x+\frac{1}{N} \operatorname{meas}(\Omega) .
\end{aligned}
$$

By the inequalities above, thanks to (4.6), we obtain

$$
\begin{aligned}
\tau \sum_{i=1}^{n} \int_{\left\{\left|U^{i}\right| \leq k\right\}}\left|\nabla U^{i}\right|^{p_{-}} d x & \leq k C\left(u_{0}, f\right)+\frac{n}{N} \operatorname{meas}(\Omega) \\
& \leq k C\left(u_{0}, f\right)+\operatorname{meas}(\Omega) \leq k\left(C\left(u_{0}, f\right)+\operatorname{meas}(\Omega)\right)
\end{aligned}
$$

for all $k \geq 1$.

\section{Convergence and existence result}

In this section, we prove the existence of an entropy solution of problem $(P)$. First of all, we introduce the appropriate spaces for the entropy formulation of the nonlinear parabolic problem $(P)$.

We define the space:

$$
V=\left\{v \in L^{p_{-}}\left(0, T ; W^{1, p(\cdot)}(\Omega)\right): \nabla v \in\left(L^{p(\cdot)}\left(Q_{T}\right)\right)^{d}\right\}
$$

and

$$
\begin{aligned}
\mathcal{T}^{1, p(\cdot)}\left(Q_{T}\right)= & \left\{u: \Omega \times(0, T] ; \text { measurable } \mid T_{k}(u) \in L^{p_{-}}\left(0, T ; W^{1, p(\cdot)}(\Omega)\right)\right. \\
& \text { with } \left.\nabla T_{k}(u) \in\left(L^{p(\cdot)}\left(Q_{T}\right)\right)^{d} \text { for every } k>0\right\} .
\end{aligned}
$$


Definition 5.1. An entropy solution to problem $(P)$ is a function $u \in \mathcal{T}^{1, p(\cdot)}\left(Q_{T}\right) \cap C\left(0, T ; L^{1}(\Omega)\right)$ such that and for all $k>0$ we have

$$
\begin{aligned}
& \int_{0}^{t} \int_{\Omega} a(x, \nabla u) \nabla T_{k}(u-\varphi)+\int_{0}^{t} \int_{\Omega} b(u) T_{k}(u-\varphi) \\
& \leq-\int_{0}^{t}\left\langle\frac{\partial \varphi}{\partial s}, T_{k}(u-\varphi)\right\rangle+\int_{\Omega} J_{k}(u(0)-\varphi(0))-\int_{\Omega} J_{k}(u(t)-\varphi(t)) \\
& +\int_{0}^{t} \int_{\Omega} f T_{k}(u-\varphi)
\end{aligned}
$$

for all $\varphi \in L^{\infty}(Q) \cap V \cap W^{1,1}\left(0, T ; L^{1}(\Omega)\right)$ and $t \in[0, T]$.

Our main result is

Theorem 5.2. Let hypotheses $(H 1)-(H 3)$ be satisfied. Then the nonlinear parabolic problem $(P)$ has an entropy solution.

Proof. The proof is divided into two steps

Step 1: The Rothe function. We introduce a piecewise linear extension:

$$
\left\{\begin{array}{l}
u^{N}(0):=u_{0} \\
u^{N}(t):=U^{n-1}+\left(U^{n}-U^{n-1}\right) \frac{t-t^{n-1}}{\tau}
\end{array}\right.
$$

for all $\left.t \in] t^{n-1}, t^{n}\right], n=1, \cdots, N$, in $\Omega$ and a piecewise constant function

$$
\left\{\begin{array}{l}
\bar{u}^{N}(0):=u_{0}, \\
\left.\left.\bar{u}^{N}(t):=U^{n}, \forall t \in\right] t^{n-1}, t^{n}\right], n=1, \cdots, N \text {, in } \Omega,
\end{array}\right.
$$

where $t^{n}:=n \tau$ and $\left(U^{n}\right)_{1 \leq n \leq N}$ an entropy solution of $\left(P_{n}\right)$.

By Theorem 3.3, for any $N \in \mathbb{N}$; the solution $\left(U^{n}\right)_{N \in \mathbb{N}}$ of problems $\left(P_{n}\right)$ is unique. Thus, $u^{N}$ and $\bar{u}^{N}$ are uniquely defined. Consequently, by the Theorem 4.1, we deduce the existence of a constant $C\left(T, u_{0}, f\right)$ not depending on $N$ such that for all $N \in \mathbb{N}$, we have

$$
\begin{aligned}
& \left\|\bar{u}^{N}-u^{N}\right\|_{L^{1}\left(Q_{T}\right)} \leq \frac{1}{N} C\left(T, u_{0}, f\right) \\
& \left\|u^{N}\right\|_{L^{1}\left(Q_{T}\right)} \leq C\left(T, u_{0}, f\right) \\
& \left\|\bar{u}^{N}\right\|_{L^{1}\left(Q_{T}\right)} \leq C\left(T, u_{0}, f\right) \\
& \left\|\frac{\partial u^{N}}{\partial t}\right\|_{L^{1}\left(Q_{T}\right)} \leq C\left(T, u_{0}, f\right) \\
& \left\|b\left(\bar{u}^{N}\right)\right\|_{L^{1}\left(Q_{T}\right)} \leq C\left(T, u_{0}, f\right)
\end{aligned}
$$


Moreover combining Proposition 2.1 and Young inequality, we get

$$
\begin{aligned}
\left\|\nabla T_{k}\left(U^{N}\right)\right\|_{p(x)}^{p_{-}} & \leq \max \left\{\rho_{p(x)}\left(\nabla T_{k}\left(U^{N}\right)\right), \rho_{1, p(x)}\left(\nabla T_{k} U^{N}\right)^{\frac{p_{-}}{p_{+}}}\right\} \\
& \leq \rho_{p(x)}\left(\nabla T_{k}\left(U^{N}\right)\right)+\rho_{1, p(x)}\left(\nabla T_{k} U^{N}\right)^{\frac{p_{-}}{p_{+}}} \\
& \leq \rho_{p(x)}\left(\nabla T_{k}\left(U^{N}\right)\right)+\frac{p_{-}}{p_{+}} \rho_{p(x)}\left(\nabla T_{k}\left(U^{N}\right)\right)+1-\frac{p_{-}}{p_{+}} \\
& \leq 2 \rho_{p(x)}\left(\nabla T_{k}\left(U^{N}\right)\right)+1 .
\end{aligned}
$$

Thanks to Poincaré-Wirtinger inequality, we have

$$
\left\|T_{k}\left(U^{N}\right)\right\|_{p(x)} \leq C \operatorname{meas}(\Omega)\left\|\nabla T_{k}\left(U^{N}\right)\right\|_{p(x)}+k\|1\|_{p(x)}
$$

which implies that

$$
\left\|T_{k}\left(U^{N}\right)\right\|_{p(x)}^{p_{-}} \leq 2^{p_{-}-1}\left((C \operatorname{meas}(\Omega))^{p_{-}}\left\|\nabla T_{k}\left(U^{N}\right)\right\|_{p(x)}^{p_{-}}+k^{p_{-}}\|1\|_{p(x)}^{p_{-}}\right)
$$

then it follows that,

$$
\begin{aligned}
\left\|T_{k}\left(U^{N}\right)\right\|_{1, p(x)}^{p_{-}} \leq & 2^{p_{-}-1}\left[(C \operatorname{meas}(\Omega))^{p_{-}}\left(2 \rho_{p(x)}\left(\nabla T_{k}\left(U^{N}\right)\right)+1\right)+k^{p_{-}}\|1\|_{p(x)}^{p_{-}}\right] \\
& +2 \rho_{p(x)}\left(\nabla T_{k}\left(U^{N}\right)\right)+1 .
\end{aligned}
$$

Therefore,

$$
\begin{aligned}
\int_{0}^{T}\left\|T_{k}\left(U^{N}\right)\right\|_{1, p(.)}^{p_{-}} d t \leq & 2^{p_{-}-1}\left[(C \operatorname{meas}(\Omega))^{p_{-}}\left(2 \int_{0}^{T} \rho_{p(.)}\left(\nabla T_{k}\left(U^{N}\right)\right) d t+T\right)\right. \\
& \left.+T k^{p_{-}}\|1\|_{p(x)}^{p_{-}}\right]+2 \int_{0}^{T} \rho_{p(.)}\left(\nabla T_{k}\left(U^{N}\right)\right) d t+T \\
\leq & 2^{p_{-}-1}\left[(C \operatorname{meas}(\Omega))^{p_{-}}\left(2 \sum_{n=1}^{N} \int_{(n-1) \tau}^{n \tau} \rho_{p(.)}\left(\nabla T_{k}\left(U^{N}\right)\right) d t+T\right)\right. \\
& \left.+T k^{p_{-}}\|1\|_{p(.)}^{p_{-}}\right]+2 \sum_{n=1}^{N} \int_{(n-1) \tau}^{n \tau} \rho_{p(.)}\left(\nabla T_{k}\left(U^{N}\right)\right) d t+T \\
\leq & 2^{p_{-}-1}\left[(C \operatorname{meas}(\Omega))^{p_{-}}\left(2 \sum_{n=1}^{N} \tau \rho_{p(.)}\left(\nabla T_{k}\left(U^{n}\right)\right)+T\right)\right. \\
& \left.+T k^{p_{-}}\|1\|_{p(.)}^{p_{-}}\right]+2 \sum_{n=1}^{N} \tau \rho_{1, p(.)}\left(T_{k}\left(U^{n}\right)\right)+T .
\end{aligned}
$$

Consequently from stability result 4 it follows that

$$
\left\|T_{k}\left(\bar{u}^{N}\right)\right\|_{L^{p_{-}\left(0, T ; W^{1, p(x)}(\Omega)\right)}} \leq C\left(T, k, u_{0}, f, p_{-}\right) .
$$

Lemma 5.3. Let hypotheses $(1.3)-(1.5)$ be satisfied. Then the sequence $\left(\bar{u}^{N}\right)_{N \in \mathbb{N}}$ converges in measure and a.e. in $Q_{T}$. 
Proof. Let $\varepsilon, r, k$ be positive numbers. For $N, M \in \mathbb{N}$, we have the inclusion

$$
\begin{aligned}
\left\{\left|\bar{u}^{N}-\bar{u}^{M}\right|>r\right\} \subset & \quad\left\{\left|\bar{u}^{N}\right|>k\right\} \cup\left\{\left|\bar{u}^{M}\right|>k\right\} \\
\cup & \left\{\left|\bar{u}^{N}\right| \leq k,\left|\bar{u}^{M}\right| \leq k,\left|\bar{u}^{N}-\bar{u}^{M}\right|>r\right\} .
\end{aligned}
$$

Firstly, we have

$$
\text { meas }\left\{\left|\bar{u}^{N}\right|>k\right\} \leq \frac{1}{k}\left\|\bar{u}^{N}\right\|_{L^{1}\left(Q_{T}\right)} \leq \frac{1}{k} C\left(T, u_{0}, f\right) .
$$

Similarly, we have

$$
\text { meas }\left\{\left|\bar{u}^{M}\right|>k\right\} \leq \frac{1}{k}\left\|\bar{u}^{N}\right\|_{L^{1}\left(Q_{T}\right)} \leq \frac{1}{k} C\left(T, u_{0}, f\right) .
$$

Therefore, for $k$ large enough, we have

$$
\operatorname{meas}\left(\left\{\left|\bar{u}^{M}\right|>k\right\} \cup\left\{\left|\bar{u}^{M}\right|>k\right\}\right) \leq \frac{\varepsilon}{2} .
$$

Secondly, by the Proposition 2.1 and Young inequality, we have

$$
\begin{aligned}
\left\|\nabla T_{k}\left(\bar{u}^{N}\right)\right\|_{L^{p(.)}\left(Q_{T}\right)} & \leq \max \left\{\left(\int_{0}^{T} \int_{\Omega}\left|\nabla T_{k}\left(\bar{u}^{N}\right)\right|^{p(x)} d x d t\right)^{\frac{1}{p_{-}}} ;\left(\int_{0}^{T} \int_{\Omega}\left|\nabla T_{k}\left(\bar{u}^{N}\right)\right|^{p(x)} d x d t\right)^{\frac{1}{p_{+}}}\right\} \\
& \leq\left(\int_{0}^{T} \int_{\Omega}\left|\nabla T_{k}\left(\bar{u}^{N}\right)\right|^{p(x)} d x d t\right)^{\frac{1}{p_{-}}}+\left(\int_{0}^{T} \int_{\Omega}\left|\nabla T_{k}\left(\bar{u}^{N}\right)\right|^{p(x)} d x d t\right)^{\frac{1}{p_{+}}}
\end{aligned}
$$

and also, we have

$$
\begin{aligned}
\int_{0}^{T} \int_{\Omega}\left|\nabla T_{k}\left(\bar{u}^{N}\right)\right|^{p(x)} d x d t=\int_{0}^{T} \rho_{p(.)}\left(T_{k}\left(\nabla \bar{u}^{N}\right)\right) & =\sum_{n=1}^{N} \int_{(n-1) \tau}^{n \tau} \rho_{p(.)}\left(\nabla T_{k}\left(U^{N}\right)\right) d t \\
& \leq \sum_{n=1}^{N} \tau \rho_{p(.)}\left(\nabla T_{k}\left(U^{n}\right)\right) .
\end{aligned}
$$

Therefore, using the stability result 4 and Proposition 2.1, it follows

$$
\left\|\nabla T_{k}\left(\bar{u}^{N}\right)\right\|_{\left(L^{p(x)}\left(Q_{T}\right)\right)^{d}} \leq\left(k C\left(u_{0}, f\right)\right)^{\frac{1}{p_{-}}}+\left(k C\left(u_{0}, f\right)\right)^{\frac{1}{p_{+}}} .
$$

Since by the Poincaré-Wirtinger inequality, we have

$$
\left\|T_{k}\left(\bar{u}^{N}\right)\right\|_{L^{p(x)}\left(Q_{T}\right)} \leq C \operatorname{meas}(\Omega)\left\|\nabla T_{k}\left(\bar{u}^{N}\right)\right\|_{L^{p(x)}\left(Q_{T}\right)}+k\|1\|_{L^{p(x)}\left(Q_{T}\right)},
$$

then by (5.12), we get

$$
\left\|T_{k}\left(\bar{u}^{N}\right)\right\|_{L^{p(x)}\left(Q_{T}\right)} \leq C \operatorname{meas}(\Omega)\left(\left(k C\left(u_{0}, f\right)\right)^{\frac{1}{p_{-}}}+\left(k C\left(u_{0}, f\right)\right)^{\frac{1}{p_{+}}}\right)+\left.k\|1\|\right|_{L^{p(x)}\left(Q_{T}\right)} .
$$

Hence, the sequences $\left(T_{k}\left(\bar{u}^{N}\right)\right)_{N \in \mathbb{N}}$ are bounded in $L^{p(\cdot)}\left(Q_{T}\right)$. Then, there exists a subsequence, still denoted by $\left(T_{k}\left(\bar{u}^{N}\right)\right)_{N \in \mathbb{N}}$, that is a Cauchy sequence in $L^{p(.)}\left(Q_{T}\right)$ and in measure. Thus, there exists $N_{0} \in \mathbb{N}$ such that for all $N, M \geq N_{0}$, we have

$$
\text { meas }\left(\left\{\left|\bar{u}^{N}\right| \leq k,\left|\bar{u}^{M}\right| \leq k,\left|\bar{u}^{N}-\bar{u}^{M}\right|>r\right\}\right)<\frac{\varepsilon}{2} .
$$

Then, by (5.11) and (5.14), $\left(\bar{u}^{N}\right)_{N \in \mathbb{N}}$ converges in measure. Therefore there exists an element $u \in M\left(Q_{T}\right)$ such that

$$
\bar{u}^{N} \rightarrow u \text { a.e. } \operatorname{in} Q_{T} .
$$


Now, by $(5.12)$

$$
\left(\nabla T_{k}\left(\bar{u}^{N}\right)\right)_{N \in \mathbb{N}} \text { is uniformly bounded in, }\left(L^{p(\cdot)}\left(Q_{T}\right)\right)^{d} .
$$

Hence there exists a subsequence, still denoted by

$$
\left(\nabla T_{k}\left(\bar{u}^{N}\right)\right)_{N \in \mathbb{N}} \text { converges weakly to an element } V \text { in } L^{p(.)}\left(Q_{T}\right) .
$$

Since

$$
T_{k}\left(\bar{u}^{N}\right) \text { converges weakly to } T_{k}(u) \text { in } L^{p(.)}\left(Q_{T}\right) \text {. }
$$

Then

$$
\nabla T_{k}\left(\bar{u}^{N}\right) \text { converges weakly to } \nabla T_{k}(u) \text { in }\left(L^{p(.)}\left(Q_{T}\right)\right)^{d} .
$$

and by (5.8) we conclude that

$$
T_{k}(u) \in L^{p_{-}}\left(0, T ; W^{1, p(.)}(\Omega)\right) \text { for all } k>0 .
$$

In the sequel, we need the following Lemma (see [22]).

Lemma 5.4. Let $\left(v_{n}\right)_{n>1}$ be a sequence of measurable functions in $\Omega$. If $\left(v_{n}\right)_{n>1}$ converges in measure to $v$ and is uniformly bounded in $L^{p(.)}(\Omega)$ for some $1<<p(.) \in L^{\infty}(\Omega)$, then $\left(v_{n}\right)_{n \geq 1} \rightarrow v$ strongly in $L^{1}(\Omega)$.

Now, we have the following result

Lemma 5.5. Let hypotheses (1.3) - (1.5) be satisfied. Then

(i) $\left(\nabla T_{k}\left(\bar{u}^{N}\right)\right)_{N \in \mathbb{N}}$ converges in measure to $\nabla T_{k}(u)$;

(ii) $\left(a\left(x, T_{k}\left(\bar{u}^{N}\right)\right)\right)_{N \in \mathbb{N}}$ converges strongly to $a\left(x, \nabla T_{k}(u)\right)$ in $\left(L^{1}\left(Q_{T}\right)\right)^{d}$ and weakly in $\left(L^{p^{(\cdot)}}\left(Q_{T}\right)\right)^{d}$.

Proof. (i) Let $h \geq 1$, from the Hölder type inequality, we have

$$
\begin{aligned}
\operatorname{meas}\left\{\left|\nabla T_{k}\left(\bar{u}^{N}\right)-\nabla T_{k}(u)\right|>h\right\} & \leq \frac{1}{h} \int_{Q_{T}}\left|\nabla T_{k}\left(\bar{u}^{N}\right)-\nabla T_{k}(u)\right| d x d s \\
& \leq \frac{1}{h}\left(\frac{1}{p_{-}}+\frac{1}{p_{+}}\right)\left\|\nabla T_{k}\left(\bar{u}^{N}\right)-\nabla T_{k}(u)\right\|_{p(.)}\|1\|_{p^{\prime}(.)} \\
& \leq \frac{1}{h}\left(\frac{1}{p_{-}}+\frac{1}{\left(p_{-}\right)^{\prime}}\right)\left(\left\|\nabla T_{k}\left(\bar{u}^{N}\right)\right\|_{p(.)}+\left\|\nabla T_{k}(u)\right\|_{p(.)}\right)\|1\|_{p^{\prime}(.)} .
\end{aligned}
$$

So by (5.15), meas $\left\{\left|\nabla T_{k}\left(\bar{u}^{N}\right)-\nabla T_{k}(u)\right|>h\right\} \rightarrow 0$ as $h \rightarrow \infty$ for any fixed $k>0$ and the proof of $(i)$ is complete.

As a consequence of $(i)$, up to a subsequence, we can assume that $\nabla T_{k}\left(\bar{u}^{N}\right) \rightarrow \nabla T_{k}(u)$ a.e in $Q_{T}$.

(ii) Since $a(x, \xi)$ is continuous with respect to $\xi \in \mathbb{R}^{N}$, then by $(i)$ we deduce that

$$
\left(a\left(x, T_{k}\left(\bar{u}^{N}\right)\right)\right)_{N \in \mathbb{N}} \text { converges in measure to } a\left(x, \nabla T_{k}(u)\right) \text { and a.e. in } Q_{T} \text {. }
$$


Moreover, using the hypotheses (1.3) and (5.12) one shows that $\left(a\left(x, \nabla T_{k}\left(\bar{u}^{N}\right)\right)\right)_{N \in \mathbb{N}}$ is uniformly bounded in $\left(L^{p^{\prime}(\cdot)}\left(Q_{T}\right)\right)^{d}$.

Consequently, in the one part thanks to Lemma 5.4 it follows that $\left(a\left(x, T_{k}\left(\bar{u}^{N}\right)\right)\right)_{N \in \mathbb{N}} \rightarrow a\left(x, \nabla T_{k}(u)\right)$ strongly in $\left(L^{1}\left(Q_{T}\right)\right)^{d}$.

On the other part, we can extract a subsequence still denoted by $\left(a\left(x, \nabla T_{k}\left(\bar{u}^{N}\right)\right)\right)_{N \in \mathbb{N}}$ such that $a\left(x, \nabla T_{k}\left(\bar{u}^{N}\right)\right) \rightarrow \zeta_{k}$ in $\left(L^{p^{\prime}(.)}\left(Q_{T}\right)\right)^{d}$. Since each of the convergence implies the weak $L^{1}$ convergence, $\zeta_{k}$ can be identified with $a\left(x, \nabla T_{k}(u)\right)$, thus $a\left(x, \nabla T_{k}(u)\right) \in\left(L^{p^{\prime}(\cdot)}\left(Q_{T}\right)\right)^{d}$. This completes the proof.

Lemma 5.6. $\left(\bar{u}^{N}\right)_{N \in \mathbb{N}}$ converges a.e. in $\Sigma_{T}$.

Proof. We know that the trace operator is compact from $W^{1,1}(\Omega)$ into $L^{1}(\partial \Omega)$, then there exists a constant $C$ such that

$$
\int_{0}^{T}\left\|T_{k}\left(\bar{u}^{N}(t)\right)-T_{k}(u(t))\right\|_{L^{1}(\partial \Omega)} d t \leq C \int_{0}^{T}\left\|T_{k}\left(\bar{u}^{N}(t)\right)-T_{k}(u(t))\right\|_{W^{1,1}(\Omega)} d t .
$$

Since $W^{1, p(.)}(\Omega) \hookrightarrow W^{1,1}(\Omega)$ for all $p() \geq$.1 , then by the Hölder type inequality, we deduce that

$$
T_{k}\left(\bar{u}^{N}(t)\right) \rightarrow T_{k}(u) \text { in } L^{1}\left(\Sigma_{T}\right) \text { and a.e. on } \Sigma_{T} .
$$

So, there exists $A \subset \Sigma_{T}$ such that $T_{k}\left(\bar{u}^{N}(t)\right)$ converges to $T_{k}(u(t))$ on $\Sigma_{T} \backslash A$ with meas $(A)=0$. For every $k>0$, we set

$$
A_{k}=\left\{(t, x) \in \Sigma_{T}:\left|T_{k}(u(t))\right|<k\right\}, \quad \text { and } B=\Sigma_{T} \backslash \bigcup_{k=1}^{\infty} A_{k} .
$$

We have, by Hölder's inequality

$$
\begin{aligned}
\operatorname{meas}(B) & \leq \frac{1}{k} \int_{B}\left|T_{k}(u)\right| d \sigma \\
& \leq \frac{1}{k} \int_{0}^{T}\left\|T_{k}(u)\right\|_{L^{1}(\partial \Omega)} d t \\
& \leq \frac{1}{k} \int_{0}^{T}\left\|T_{k}(u)\right\|_{W^{1,1}(\Omega)} d t \\
& \leq \frac{1}{k} \int_{0}^{T} \int_{\Omega}\left(\left|T_{k}(u)\right|+\left|\nabla T_{k}(u)\right|\right) \\
& \leq \frac{1}{k}\left(\frac{1}{p_{-}}+\frac{1}{\left(p_{-}\right)^{\prime}}\right)\|1\|_{L^{p^{\prime}(x)}\left(Q_{T}\right)}\left(\left\|T_{k}(u)\right\|_{L^{p(x)}\left(Q_{T}\right)}+\left\|\nabla T_{k}(u)\right\|_{\left(L^{p(x)}\left(Q_{T}\right)\right)^{d}}\right) .
\end{aligned}
$$

Thanks to (5.12) and (5.13), for all $k>0$, we have

$$
\begin{aligned}
\left\|T_{k}\left(\bar{u}^{N}\right)\right\|_{L^{p(x)}(Q)}+\left\|\nabla T_{k}\left(\bar{u}^{N}\right)\right\|_{\left(L^{p(x)}(Q)\right)^{d}} \leq & 2\left(k^{\frac{1}{p_{-}}}+k^{\frac{1}{p_{+}}}\right) \\
& \times \max \left\{C\left(u_{0}, p_{+}, f, g\right)^{\frac{1}{p_{+}}}, C\left(u_{0}, p_{+}, f, g\right)^{\frac{1}{p_{+}}}\right\}
\end{aligned}
$$


We now use the Fatou's lemma in (5.19) to get

$$
\begin{aligned}
\left\|T_{k}(u)\right\|_{L^{p(x)}(Q)}+\left\|\nabla T_{k}(u)\right\|_{\left(L^{p(x)}(Q)\right)^{d}} \leq & 2\left(k^{\frac{1}{p_{-}}}+k^{\frac{1}{p_{+}}}\right) \\
& \times \max \left\{C\left(u_{0}, p_{+}, f, g\right)^{\frac{1}{p_{+}}}, C\left(u_{0}, p_{+}, f, g\right)^{\frac{1}{p_{+}}}\right\},
\end{aligned}
$$

and (5.18) becomes

$$
\text { meas }(B) \leq 2\left(\frac{1}{k^{1-\frac{1}{p_{-}}}}+\frac{1}{k^{1-\frac{1}{p_{+}}}}\right) \max \left\{C\left(u_{0}, p_{+}, f, g\right)^{\frac{1}{p_{+}}}, C\left(u_{0}, p_{+}, f, g\right)^{\frac{1}{p_{+}}}\right\} .
$$

Therefore, we get by letting $k \rightarrow \infty$ in (5.20) that meas $(B)=0$.

Let us now define on $\partial \Omega$, the function $v$ by

$$
v(t, x)=T_{k}(u(t))(x) \text { if }(x, t) \in A_{k} .
$$

We take $(x, t) \in \Sigma_{T} \backslash(A \cup B)$; then there exists $k>0$ such that $(x, t) \in A_{k}$ and we have

$$
\bar{u}^{N}(t, x)-v(t, x)=\left(\bar{u}^{N}(t, x)-T_{k}\left(\bar{u}^{N}(t)\right)(x)\right)+\left(T_{k}\left(\bar{u}^{N}(t)\right)(x)-T_{k}(u(t))(x)\right) .
$$

Since $(x, t) \in A_{k}$, we have $\left|T_{k}\left(\bar{u}^{N}(t)\right)(x)\right|<k$ from which we deduce that $T_{k}\left(\bar{u}^{N}(t)\right)(x)=\bar{u}^{N}(t, x)$. Therefore,

$$
\bar{u}^{N}(t, x)-v(t, x)=\left(T_{k}\left(\bar{u}^{N}(t)\right)(x)-T_{k}(u(t))(x)\right) \rightarrow 0, \text { as } N \rightarrow \infty .
$$

This means that $\left(\bar{u}^{N}\right)$ converges to $v$ a.e. on $\Sigma_{T}$.

Lemma 5.7. The sequence $\left(\bar{u}^{N}\right)_{N \in \mathbb{N}}$ converges to $u$ in $C\left(0, T ; L^{1}(\Omega)\right)$.

Proof. Let $\left(t^{n}=n \tau_{N}\right)_{n=1}^{N}$ and $\left(t^{m}=m \tau_{M}\right)_{n=1}^{M}$ be two partitions of the interval $[0, T]$ and let $\left(u^{N}(t), \bar{u}^{N}(t)\right),\left(u^{M}(t) ; \bar{u}^{M}(t)\right)$; be the semi-discrete solutions defined by (5.1), (5.2) and corresponding to the respective partitions. Let $\varphi \in L^{\infty}(\Omega) \cap V \cap W^{1,1}\left(0, T ; L^{1}(\Omega)\right)$. We rewrite (3.1) in the forms

$$
\begin{aligned}
& \int_{0}^{t}\left\langle\frac{\partial u^{N}}{\partial s}, T_{k}\left(\bar{u}^{N}-\varphi\right)\right\rangle d s+\int_{0}^{t} \int_{\Omega} a\left(x, \nabla \bar{u}^{N}\right) \cdot \nabla T_{k}\left(\bar{u}^{N}-\varphi\right) d x d s \\
& +\int_{0}^{t} \int_{\Omega} b\left(\bar{u}^{N}\right) T_{k}\left(\bar{u}^{N}-\varphi\right) d x d s \\
& \leq \int_{0}^{t} \int_{\Omega} f_{N} T_{k}\left(\bar{u}^{N}-\varphi\right) d x d s
\end{aligned}
$$

and

$$
\begin{aligned}
& \int_{0}^{t}\left\langle\frac{\partial u^{M}}{\partial s}, T_{k}\left(\bar{u}^{M}-\varphi\right)\right\rangle d s+\int_{0}^{t} \int_{\Omega} a\left(x, \nabla \bar{u}^{M}\right) \cdot \nabla T_{k}\left(\bar{u}^{M}-\varphi\right) d x d s \\
& +\int_{0}^{t} \int_{\Omega} b\left(\bar{u}^{M}\right) T_{k}\left(\bar{u}^{M}-\varphi\right) d x d s \\
& \leq \int_{0}^{t} \int_{\Omega} f_{M} T_{k}\left(\bar{u}^{M}-\varphi\right) d x d s
\end{aligned}
$$


where

$$
\begin{gathered}
\left.\left.f_{N}(t, x)=f_{n}(x) \quad \forall t \in\right] t^{n-1}, t^{n}\right] \\
f_{M}(t, x)=f_{m}(x)
\end{gathered}
$$

Let $h>1$, in inequality (5.21) we take $\varphi=T_{h}\left(\bar{u}^{M}\right)$ and in inequality (5.22) we take $\varphi=T_{h}\left(\bar{u}^{N}\right)$. Summing both inequalities, we get, for $k=1$,

$$
\begin{aligned}
& \int_{0}^{t}\left\langle\frac{\partial\left(u^{N}-u^{M}\right)}{\partial s}, T_{1}\left(u^{N}-u^{M}\right)\right\rangle d s+I_{N, M}(h) \\
& +\int_{0}^{t} \int_{\Omega} b\left(\bar{u}^{N}\right) T_{1}\left(\bar{u}^{N}-T_{h}\left(\bar{u}^{M}\right)\right) d x d s \\
& +\int_{0}^{t} \int_{\Omega} b\left(\bar{u}^{M}\right) T_{1}\left(\bar{u}^{M}-T_{h}\left(\bar{u}^{N}\right)\right) d x d s \\
\leq & \int_{0}^{t}\left\langle\frac{\partial\left(u^{N}-u^{M}\right)}{\partial s}, T_{1}\left(u^{N}-u^{M}\right)\right\rangle-\left\langle\frac{\partial u^{N}}{\partial s}, T_{1}\left(\bar{u}^{N}-T_{h}\left(\bar{u}^{M}\right)\right)\right\rangle d s \\
& -\int_{0}^{t}\left\langle\frac{\partial u^{M}}{\partial s}, T_{1}\left(\bar{u}^{M}-T_{h}\left(\bar{u}^{N}\right)\right)\right\rangle d s \\
& +\int_{0}^{t} \int_{\Omega}\left[f_{N} T_{1}\left(\bar{u}^{N}-T_{h}\left(\bar{u}^{M}\right)\right)+f_{M} T_{1}\left(\bar{u}^{M}-T_{h}\left(\bar{u}^{N}\right)\right)\right] d x d s
\end{aligned}
$$

where

$$
\begin{aligned}
I_{N, M}(h)= & \int_{0}^{t} \int_{\Omega} a\left(x, \nabla \bar{u}^{N}\right) \cdot \nabla T_{1}\left(\bar{u}^{N}-T_{h}\left(\bar{u}^{M}\right)\right) d x d s \\
& +\int_{0}^{t} \int_{\Omega} a\left(x, \nabla \bar{u}^{M}\right) \cdot \nabla T_{1}\left(\bar{u}^{M}-T_{h}\left(\bar{u}^{N}\right)\right) d x d s .
\end{aligned}
$$

We have

$$
\begin{aligned}
\left|\int_{0}^{t}\left\langle\frac{\partial\left(u^{N}-u^{M}\right)}{\partial s}, T_{1}\left(u^{N}-u^{M}\right)\right\rangle d s\right| & \leq\left\|\frac{\partial\left(u^{N}-u^{M}\right)}{\partial s}\right\|_{L^{1}\left(Q_{T}\right)}\left\|T_{1}\left(u^{N}-u^{M}\right)\right\|_{L^{\infty}\left(Q_{T}\right)} \\
& \leq 2 C\left(T, f, u_{0}\right)\left\|T_{1}\left(u^{N}-u^{M}\right)\right\|_{L^{\infty}\left(Q_{T}\right)} .
\end{aligned}
$$

Since

$$
\lim _{N, M \rightarrow \infty}\left\|T_{1}\left(u^{N}-u^{M}\right)\right\|_{L^{\infty}\left(Q_{T}\right)}=0 .
$$

Then it follows that

$$
\lim _{h \rightarrow \infty} \lim _{N, M \rightarrow \infty} \int_{0}^{t}\left\langle\frac{\partial\left(u^{N}-u^{M}\right)}{\partial s}, T_{1}\left(u^{N}-u^{M}\right)\right\rangle d s=0 .
$$

Similarly, we show that

$$
\begin{gathered}
\lim _{h \rightarrow \infty} \lim _{N, M \rightarrow \infty}\left(\int_{0}^{t}\left\langle\frac{\partial u^{N}}{\partial s}, T_{1}\left(\bar{u}^{N}-T_{h}\left(\bar{u}^{M}\right)\right)\right\rangle+\left\langle\frac{\partial u^{M}}{\partial s}, T_{1}\left(\bar{u}^{M}-T_{h}\left(\bar{u}^{N}\right)\right)\right\rangle d s\right)=0 \\
\lim _{h \rightarrow \infty} \lim _{N, M \rightarrow \infty} \int_{0}^{t} \int_{\Omega}\left[f_{N} T_{1}\left(\bar{u}^{N}-T_{h}\left(\bar{u}^{M}\right)\right)+f_{M} T_{1}\left(\bar{u}^{M}-T_{h}\left(\bar{u}^{N}\right)\right)\right] d x d s=0
\end{gathered}
$$


and

$$
\lim _{h \rightarrow \infty} \lim _{N, M \rightarrow \infty} \int_{0}^{t} \int_{\Omega} b\left(\bar{u}^{N}\right) T_{1}\left(\bar{u}^{N}-T_{h}\left(\bar{u}^{M}\right)\right) d x d s+\int_{0}^{t} \int_{\Omega} b\left(\bar{u}^{M}\right) T_{1}\left(\bar{u}^{M}-T_{h}\left(\bar{u}^{N}\right)\right) d x d s=0 .
$$

Then, letting $N, M \rightarrow \infty$ and $h \rightarrow \infty$, in (5.23)we get

$$
\lim _{h \rightarrow \infty} \lim _{N, M \rightarrow \infty} \int_{0}^{t}\left\langle\frac{\partial\left(u^{N}-u^{M}\right)}{\partial s}, T_{1}\left(u^{N}-u^{M}\right)\right\rangle d s+\lim _{h \rightarrow \infty} \lim _{N, M \rightarrow \infty} I_{N, M}(h) \leq 0 .
$$

Since

$$
\left\langle\frac{\partial v}{\partial t}, T_{k}(v)\right\rangle=\frac{d}{d t} \int_{\Omega} J_{k}(v) \text { in } L^{1}(] 0, T[),
$$

inequality (5.25) becomes

$$
\lim _{N, M \rightarrow \infty} \int_{\Omega} J_{1}\left(u^{N}(t)-u^{M}(t)\right) d x+\lim _{h \rightarrow \infty} \lim _{N, M \rightarrow \infty} I_{N, M}(h) \leq 0 .
$$

Now, we show that

$$
\lim _{h \rightarrow \infty} \lim _{N, M \rightarrow \infty} I_{N, M}(h) \geq 0 .
$$

We consider the decomposition

$$
I_{N, M}(h)=\sum_{i=1}^{4} L_{i}(h)
$$

where

$$
\begin{aligned}
& L_{i}(h)=\int_{0}^{t} \int_{\Omega_{i}(h)} a\left(x, \nabla \bar{u}^{N}\right) \cdot \nabla T_{1}\left(\bar{u}^{N}-T_{h}\left(\bar{u}^{M}\right)\right) d x d s \\
& +\int_{0}^{t} \int_{\Omega_{i}(h)} a\left(x, \nabla \bar{u}^{M}\right) \cdot \nabla T_{1}\left(\bar{u}^{M}-T_{h}\left(\bar{u}^{N}\right)\right) d x d s
\end{aligned}
$$

and

$$
\begin{array}{ll}
\Omega_{1}(h)=\left\{\left|\bar{u}^{N}\right| \leq h,\left|\bar{u}^{M}\right| \leq h\right\} & \Omega_{2}(h)=\left\{\left|\bar{u}^{N}\right| \leq h,\left|\bar{u}^{M}\right|>h\right\} \\
\Omega_{3}(h)=\left\{\left|\bar{u}^{N}\right|>h,\left|\bar{u}^{M}\right| \leq h\right\} & \Omega_{4}(h)=\left\{\left|\bar{u}^{N}\right|>h,\left|\bar{u}^{M}\right|>h\right\} .
\end{array}
$$

On the one hand, thanks to assumption (1.4) we have

$$
L_{1}(h)=\int_{0}^{t} \int_{\Omega_{1}^{1}(h)}\left[a\left(x, \nabla \bar{u}^{N}\right)-a\left(x, \nabla \bar{u}^{M}\right)\right] \cdot \nabla\left(\bar{u}^{N}-\bar{u}^{M}\right) d x d s \geq 0 .
$$

Therefore

$$
\lim _{h \rightarrow \infty} \lim _{N, M \rightarrow \infty} L_{1}(h) \geq 0 .
$$

On the other hand, we have

$$
\begin{aligned}
L_{2}(h)= & \int_{0}^{t} \int_{\Omega_{2}^{1}(h)} a\left(x, \nabla \bar{u}^{N}\right) \cdot \nabla \bar{u}^{N} d x d s \\
& +\int_{0}^{t} \int_{\Omega_{2}^{2}(h)} a\left(x, \nabla \bar{u}^{M}\right) \cdot \nabla\left(\bar{u}^{M}-\bar{u}^{N}\right) d x d s \\
\geq & -\int_{0}^{t} \int_{\Omega_{2}^{2}(h)} a\left(x, \nabla \bar{u}^{M}\right) \cdot \nabla \bar{u}^{N} d x d s,
\end{aligned}
$$


where

$$
\begin{aligned}
& \Omega_{2}^{1}(h)=\left\{\left|\bar{u}^{N}\right| \leq h,\left|\bar{u}^{M}\right|>h,\left|\bar{u}^{N}-h \operatorname{sign}\left(\bar{u}^{M}\right)\right| \leq 1\right\}, \\
& \Omega_{2}^{2}(h)=\left\{\left|\bar{u}^{N}\right| \leq h,\left|\bar{u}^{M}\right|>h,\left|\bar{u}^{N}-\bar{u}^{M}\right| \leq 1\right\} .
\end{aligned}
$$

Now, taking $\varphi=T_{h}\left(\bar{u}^{N}\right)$ in $(5.21)$, we deduce that

$$
\lim _{h \rightarrow \infty} \lim _{N \rightarrow \infty} \int_{0}^{t} \int_{\left\{h \leq\left|\bar{u}^{N}\right| \leq h+k\right\}} a\left(x, \nabla \bar{u}^{N}\right) \cdot \nabla \bar{u}^{N}=0 .
$$

This implies

$$
\lim _{h \rightarrow \infty} \lim _{N \rightarrow \infty} \int_{0}^{t} \int_{\left\{h \leq\left|\bar{u}^{N}\right| \leq h+k\right\}}\left|\nabla \bar{u}^{N}\right|^{p(x)}=0, \quad k>0 .
$$

By the Young inequality, we have

$$
\begin{aligned}
& \left|\int_{0}^{t} \int_{\Omega_{2}^{2}(h)} a\left(x, \nabla \bar{u}^{M}\right) \cdot \nabla \bar{u}^{N} d x d s\right| \\
\leq & \int_{0}^{t} \int_{\Omega_{2}^{2}(h)}\left|\nabla \bar{u}^{M}\right| p^{p(x)-1}\left|\nabla \bar{u}^{N}\right| d x d s \\
\leq & \int_{0}^{t} \int_{\left\{h \leq\left|\bar{u}^{M}\right| \leq h+1\right\}} \frac{1}{p^{\prime}(x)}\left|\nabla \bar{u}^{M}\right|^{p(x)} d x d s+\int_{0}^{t} \int_{\left\{h-1 \leq\left|\bar{u}^{N}\right| \leq h\right\}} \frac{1}{p(x)}\left|\nabla \bar{u}^{M}\right|^{p(x)} d x d s \\
\leq & \int_{0}^{t} \int_{\left\{h \leq\left|\bar{u}^{M}\right| \leq h+1\right\}} \frac{1}{p_{-}^{\prime}}\left|\nabla \bar{u}^{M}\right|^{p(x)} d x d s+\int_{0}^{t} \int_{\left\{h-1 \leq\left|\bar{u}^{N}\right| \leq h\right\}} \frac{1}{p_{-}}\left|\nabla \bar{u}^{M}\right|^{p(x)} d x d s .
\end{aligned}
$$

Thus (5.27) gives

$$
\lim _{N, M \rightarrow \infty} \int_{0}^{t} \int_{0}^{t} \int_{\Omega_{2}^{2}(h)} a\left(x, \nabla \bar{u}^{M}\right) \cdot \nabla \bar{u}^{N} d x d s=0
$$

which implies that

$$
\lim _{h \rightarrow \infty} \lim _{N, M \rightarrow \infty} L_{2}(h) \geq 0
$$

Similarly, we show that

$$
\lim _{h \rightarrow \infty} \lim _{N, M \rightarrow \infty}\left(L_{3}(h)+L_{4}(h)\right) \geq 0 .
$$

Therefore

$$
\lim _{h \rightarrow \infty} \lim _{N, M \rightarrow \infty} I_{N, M}(h) \geq 0 .
$$

Thus (5.26) becomes

$$
\lim _{N, M \rightarrow \infty} \int_{\Omega} J_{1}\left(u^{N}(t)-u^{M}(t)\right) d x=0 .
$$

Since

$\frac{1}{2} \int_{\left\{\left|u^{N}-u^{M}\right| \leq 1\right\}}\left|u^{N}(t)-u^{M}(t)\right|^{2} d x+\int_{\left\{\left|u^{N}-u^{M}\right| \geq 1\right\}}\left|u^{N}(t)-u^{M}(t)\right| d x \leq \int_{\Omega} J_{1}\left(u^{N}(t)-u^{M}(t)\right) ;$ 
we have

$$
\begin{aligned}
& \int_{\left\{\left|u^{N}-u^{M}\right| \geq 1\right\}}\left|u^{N}(t)-u^{M}(t)\right| d x \\
= & \int_{\left\{\left|u^{N}-u^{M}\right| \leq 1\right\}}\left|u^{N}(t)-u^{M}(t)\right| d x+\int_{\left\{\left|u^{N}-u^{M}\right| \geq 1\right\}}\left|u^{N}(t)-u^{M}(t)\right| d x \\
\leq & C_{\Omega}\left(\int_{\left\{\left|u^{N}-u^{M}\right| \leq 1\right\}}\left|u^{N}(t)-u^{M}(t)\right|^{2} d x\right)^{\frac{1}{2}}+\int_{\left\{\left|u^{N}-u^{M}\right| \geq 1\right\}}\left|u^{N}(t)-u^{M}(t)\right| d x \\
\leq & C_{2}(\Omega)\left(\int_{\Omega} J_{1}\left(u^{N}(t)-u^{M}(t)\right) d x\right)^{\frac{1}{2}}+\int_{\Omega} J_{1}\left(u^{N}(t)-u^{M}(t)\right) d x .
\end{aligned}
$$

By (5.26), we deduce that $\left(u^{N}\right)_{N \in \mathbb{N}}$ is a Cauchy sequence in $C\left(0, T ; L^{1}(\Omega)\right)$. Hence $\left(u^{N}\right)_{N \in \mathbb{N}}$ converges to $\mathrm{u}$ in $C\left(0, T ; L^{1}(\Omega)\right)$.

Step 2: Existence of entropy solution. Now, we prove that the limit function $u$ is an entropy solution of the problem $(P)$. Since $u^{N}(0)=U^{0}=u_{0}$ for all $N \in \mathbb{N}$, we have $u(0,)=.u_{0}$, and inequality (5.21) implies

$$
\begin{aligned}
& \int_{0}^{t}\left\langle\frac{\partial u^{N}}{\partial s}, T_{k}\left(\bar{u}^{N}-\varphi\right)-T_{k}\left(u^{N}-\varphi\right)\right\rangle d s+\int_{0}^{t} \int_{\Omega} a\left(x, \nabla \bar{u}^{N}\right) \cdot \nabla T_{k}\left(\bar{u}^{N}-\varphi\right) d x d s \\
& +\int_{0}^{t} \int_{\Omega} b\left(\bar{u}^{N}\right) T_{k}\left(\bar{u}^{N}-\varphi\right) d x d s \\
& \leq \int_{0}^{t}\left\langle\frac{\varphi}{\partial s}, T_{k}\left(u^{N}-\varphi\right)-T_{k}\left(u^{N}-\varphi\right)\right\rangle d s+\int_{\Omega} J_{k}\left(u^{N}(0)-\varphi(0)\right) d x-\int_{\Omega} J_{k}\left(u^{N}(t)-\varphi(t)\right) d x \\
& +\int_{0}^{t} \int_{\Omega} f_{N} T_{k}\left(\bar{u}^{N}-\varphi\right) d x d s .
\end{aligned}
$$

Let $\bar{k}=k+\|\varphi\|_{\infty}$. Then

$$
\begin{aligned}
\int_{0}^{t} \int_{\Omega} a\left(x, \nabla \bar{u}^{N}\right) \cdot \nabla T_{k}\left(\bar{u}^{N}-\varphi\right) d x d s= & \int_{0}^{t} \int_{\Omega} a\left(x, \nabla T_{\bar{k}}\left(\bar{u}^{N}\right)\right) \cdot \nabla T_{k}\left(T_{\bar{k}}\left(\bar{u}^{N}\right)-\varphi\right) d x d s \\
= & \int_{0}^{t} \int_{\Omega}\left[a\left(x, \nabla T_{\bar{k}}\left(\bar{u}^{N}\right)\right) \cdot \nabla T_{\bar{k}}\left(\bar{u}^{N}\right)\right. \\
& \left.-a\left(x, \nabla T_{\bar{k}}\left(\bar{u}^{N}\right)\right) \cdot \nabla \varphi\right] \mathbf{1}_{Q(N, k)} d x d s,
\end{aligned}
$$

where $Q(N, k)=\left\{\left|T_{\bar{k}}\left(\bar{u}^{N}\right)-\varphi\right| \leq k\right\}$. Thus, the inequality (5.29) becomes

$$
\begin{aligned}
& \int_{0}^{t}\left\langle\frac{\partial u^{N}}{\partial s}, T_{k}\left(\bar{u}^{N}-\varphi\right)-T_{k}\left(u^{N}-\varphi\right)\right\rangle d s-\int_{0}^{t} \int_{\Omega} a\left(x, \nabla T_{\bar{k}}\left(\bar{u}^{N}\right)\right) \cdot \nabla \varphi \mathbf{1}_{Q(N, k)} \\
& +\int_{0}^{t} \int_{\Omega}\left[a\left(x, \nabla T_{\bar{k}}\left(\bar{u}^{N}\right)\right) \cdot \nabla T_{\bar{k}}\left(\bar{u}^{N}\right)\right] \mathbf{1}_{Q(N, k)}+\int_{0}^{t} \int_{\Omega} b\left(\bar{u}^{N}\right) T_{k}\left(\bar{u}^{N}-\varphi\right) d x d s \\
\leq & -\int_{0}^{t}\left\langle\frac{\partial \varphi}{\partial s}, T_{k}\left(u^{N}-\varphi\right)\right\rangle d s+\int_{\Omega} J_{k}\left(u^{N}(0)-\varphi(0)\right) d x-\int_{\Omega} J_{k}\left(u^{N}(t)-\varphi(t)\right) d x \\
& +\int_{0}^{t} \int_{\Omega} f_{N} T_{k}\left(\bar{u}^{N}-\varphi\right) d x d s .
\end{aligned}
$$


On the one hand, thanks to Lemma $5.5 a\left(x, \nabla T_{\bar{k}}\left(\bar{u}^{N}\right)\right)$ converges weakly to $a\left(x, \nabla T_{\bar{k}}(u)\right)$ in $\left(L^{p^{\prime}(.)}(\Omega)\right)^{d}$. Therefore,

$$
\lim _{N \rightarrow \infty} \int_{0}^{t} \int_{\Omega} a\left(x, \nabla T_{\bar{k}}\left(\bar{u}^{N}\right)\right) \cdot \nabla \varphi \mathbf{1}_{Q(N, k)}=\int_{0}^{t} \int_{\Omega} a\left(x, \nabla T_{\bar{k}}(u)\right) . \nabla \varphi \mathbf{1}_{Q(k)},
$$

where $Q(k)=\left\{\left|T_{\bar{k}}(u)-\varphi\right| \leq k\right\}$. Moreover, $a\left(x, \nabla T_{\bar{k}}\left(\bar{u}^{N}\right)\right) . \nabla T_{\bar{k}}\left(\bar{u}^{N}\right)$ is nonnegative and converges a.e. in $Q_{T}$ to $a\left(x, \nabla T_{\bar{k}}(u)\right) . \nabla T_{\bar{k}}(u)$ (see Lemma 5.5). Therefore by Fatou's lemma, we obtain

$\liminf _{N \rightarrow \infty} \int_{0}^{t} \int_{0}^{t} \int_{\Omega}\left[a\left(x, \nabla T_{\bar{k}}\left(\bar{u}^{N}\right)\right) \cdot \nabla T_{\bar{k}}\left(\bar{u}^{N}\right)\right] \mathbf{1}_{Q(N, k)} d x d s \geq \int_{0}^{t} \int_{0}^{t} \int_{\Omega}\left[a\left(x, \nabla T_{\bar{k}}(u)\right) . \nabla T_{\bar{k}}(u)\right] \mathbf{1}_{Q(k)} d x d s$.

For the fourth term of (5.30), we have

$\int_{0}^{t} \int_{\Omega} b\left(\bar{u}^{N}\right) T_{k}\left(\bar{u}^{N}-\varphi\right) d x d s=\int_{0}^{t} \int_{\Omega}\left(b\left(\bar{u}^{N}\right)-b(\varphi)\right) T_{k}\left(\bar{u}^{N}-\varphi\right) d x d s+\int_{0}^{t} \int_{\Omega} b(\varphi) T_{k}\left(\bar{u}^{N}-\varphi\right) d x d s$.

The quantity $\left(b\left(\bar{u}^{N}\right)-b(\varphi)\right) T_{k}\left(\bar{u}^{N}-\varphi\right)$ is is nonnegative and since for all $s \in \mathbb{R}, s \mapsto b(s)$ is continuous, we obtain

$$
\left(b\left(\bar{u}^{N}\right)-b(\varphi)\right) T_{k}\left(\bar{u}^{N}-\varphi\right) \rightarrow(b(u)-b(\varphi)) T_{k}\left(\bar{u}^{N}-\varphi\right) \quad \text { a.e. in } \Omega .
$$

Then, it follows by Fatou's lemma that

$$
\liminf _{N \rightarrow \infty} \int_{0}^{t} \int_{\Omega}\left(b\left(\bar{u}^{N}\right)-b(\varphi)\right) T_{k}\left(\bar{u}^{N}-\varphi\right) d x d s \geq \int_{0}^{t} \int_{\Omega}(b(u)-b(\varphi)) T_{k}(u-\varphi) d x d s .
$$

We have $b(\varphi) \in L^{1}\left(Q_{T}\right)$. Since $T_{k}\left(\bar{u}^{N}-\varphi\right)$ converges weakly-* to $T_{k}(u-\varphi)$ and $b(\varphi) \in L^{1}\left(Q_{T}\right)$, it follows that

$$
\liminf _{N \rightarrow \infty} \int_{0}^{t} \int_{\Omega} b(\varphi) T_{k}\left(\bar{u}^{N}-\varphi\right) d x d s \geq \int_{0}^{t} \int_{\Omega} b(\varphi) T_{k}(u-\varphi) d x d s .
$$

By Lemma 5.7, we deduce that $u^{N}(t) \rightarrow u(t)$ in $L^{1}(\Omega)$ for all $t \in[0, T]$, which implies that

$$
\int_{\Omega} J_{k}\left(u^{N}(t)-\varphi(t)\right) d x \rightarrow \int_{\Omega} J_{k}(u(t)-\varphi(t)) d x \quad \forall t \in[0, T] .
$$

We follow the method used in the proof of equality (5.24) to show that

$$
\lim _{N \rightarrow \infty} \int_{0}^{t}\left\langle\frac{\partial u^{N}}{\partial s}, T_{k}\left(\bar{u}^{N}-\varphi\right)-T_{k}\left(u^{N}-\varphi\right)\right\rangle d s=0 .
$$

Finally, letting $N \rightarrow \infty$ and using the above results, the continuity of $b$ and the facts that

$$
\begin{array}{rc}
f_{N} \rightarrow f & \text { in } L^{1}\left(Q_{T}\right), \\
T_{\bar{k}}\left(\bar{u}^{N}-\varphi\right) \rightarrow T_{\bar{k}}(u-\varphi) & \text { in } L^{\infty}\left(Q_{T}\right),
\end{array}
$$

we deduce that $u$ is an entropy solution of the nonlinear parabolic problem $(P)$. 


\section{Conclusion}

In this paper we prove the existence and uniqueness of an entropy solution for a non- linear parabolic equation with homogeneous Neumann boundary conditions and initial data in $L^{1}$ by a time discretization technique.

This method turns out to be better suited for the study of parabolic problems under Neumanntype boundary conditions. However, this technique assumes that the associated elliptic problem is well posed. This study opens up new perspectives, we could always in the context of the Sobolev space with variable exponents look at the problem with measure data or consider the function $b$ as maximal monotone graph. 


\section{References}

[1] M. Abdellaoui, E. Azroul, S. Ouaro and U. Traoré, "Nonlinear parabolic capacity and renormalized solutions for PDEs with diffuse measure data and variable exponent", An. Univ. Craiova Ser. Mat. Inform., vol. 46, no. 2, pp. 269-297, 2019.

[2] M. Abdellaoui and E. Azroul, "Nonlinear parabolic equations with data soft measure data", J. Nonlinear Evol. Equ. Appl., vol. 2019, no. 7, pp. 115-133, 2020.

[3] B. Andreianov, M. Bendahmane, K. H. Karlsenc and S. Ouaro, "Well-posedness results for triply nonlinear degenerate parabolic equations.", vol. 247, no. 1, pp. 277-302, 2009.

[4] F. Andreu, J. M. Mazón, S. Segura de Léon and J. Toledo, "Existence and uniqueness for a degenerate parabolic equation with $L^{1}$ data", Trans. Amer. Math. Soc., vol. 351, no. 1, pp. 285-306, 1999.

[5] F. Andreu, N. Igbida, J. M. Mazón and J. Toledo, " $L^{1}$ existence and uniqueness results for quasi-linear elliptic equations with nonlinear boundary conditions", Ann. Inst. H. Poincaré Anal. Non Linéaire, vol. 20, no. 1, pp. 61-89, 2017.

[6] M. Bendahmane, P. Wittbold and A. Zimmermann, "Renormalized solutions for a nonlinear parabolic equation with variable exponents and $L^{1}$ data", J. Differential Equations, vol. 249, no. 6 , pp. 1483-1515, 2010.

[7] F. Benzekri and A. El Hachimi, "Doubly nonlinear parabolic equations related to the $p$ Laplacian operator: Semi-discretization", Electron. J. Differential Equations, vol. 2003, no. 113, pp. 1-14, 2003.

[8] P. Bénilan, L. Boccardo, T. Gallouët, M. Pierre and J. L. Vazquez, "An $L^{1}$ theory of existence and uniqueness of nonlinear elliptic equations", Ann. Scuola Norm. Sup. Pisa Cl. Sci. (4), vol. 22, no. 2, pp. 214-273, 1995.

[9] B. K. Bonzy, I. Nyanquini and S. Ouaro, "Existence and uniqueness of weak and entropy solutions for homogeneous Neumann boundary-value problems involving variable exponents", Electron. J. Differential Equations, vol. 2012, no. 12, pp. 1-19, 2012.

[10] A. Dall'Aglio, "Approximated solutions of equations with $L^{1}$ data. Application to the $H$ convergence of quasi-linear parabolic equations", Ann. Mat. Pura Appl.(4), vol. 170, pp. 207$240,1996$.

[11] L. Diening, P. Harjulehto, P. Hästö and M. Růžička, Lebesgue and Sobolev spaces with variable exponents, Lecture Notes in Mathematics, vol. 2017, New York: Springer-Verlag Heidelberg, 2011. 
[12] R. J. DiPerna and P.-L. Lions, "On the Cauchy problem for Boltzmann equations: global existence and weak stability", Ann. of Math. (2), vol. 130, no. 2, pp. 321-366, 1989.

[13] A. Eden, B. Michaux and J.-M. Rakotoson, "Semi-discretized nonlinear evolution equations as discrete dynamical systems and error analysis", Indiana Univ. Math. J., vol. 39, no. 3, pp. 737-783, 1990.

[14] X. L. Fan and D. Zhao, "On the generalized Orlicz-Sobolev space $W^{k, p(x)}(\Omega)$ ", Journal of Gansu Education College, vol. 12, no. 1, pp. 1-6, 1998.

[15] G. Gagneux and M. Madaune-Tort, Analyse mathématique de modèles non linéaires de l'ingénierie pétrolière, Mathématiques et Applications 22, Berlin: Springer, 1996.

[16] A. El Hachimi and M. R. Sidi Ammi, "Thermistor problem: a nonlocal parabolic problem", in Differential Equations and Mechanics. Electron. J. Diff. Eqns., vol. 2004, no. 11, 2004, pp. $117-128$.

[17] A. El Hachimi, J. Igbida and A. Jamea, "Existence result for nonlinear parabolic problems with $L^{1}$-data", Appl. Math. (Warsaw), vol. 37, no. 4, pp. 483-508, 2010.

[18] S. Ouaro, S. Soma, "Weak and entropy solutions to nonlinear Neumann boundary valueproblems with variable exponents", Complex Var. Elliptic Equ., vol. 56, nos. 7-9, pp. 829-851, 2011.

[19] S. Ouaro, A. Tchousso, "Well-posedness result for a nonlinear elliptic problem involving variable exponent and Robin type boundary condition", Afr. Diaspora J. Math., vol. 11, no. 2, pp. 36-64, 2011.

[20] S. Ouaro and A. Ouédraogo, "Nonlinear parabolic problems with variable exponent and $L^{1}$ data", Electron. J. Differential Equations, vol. 2017, no. 32, pp. 1-32, 2017.

[21] S. Ouaro and U. Traoré, "Nonlinear parabolic problem with variable exponent and measure data", J. Nonlinear Evol. Equ. Appl., vol. 2020, no. 5, pp. 65-93, 2020.

[22] M. Sanchón and J. M. Urbano, "Entropy solution for $p(x)$-Laplace equation", Trans. Amer. Math. Soc., vol. 361, no. 2, pp. 6387-6404, 2009.

[23] H. Redwane, "Nonlinear parabolic equation with variable exponents and diffuse measure data", J. Nonl. Evol. Equ. Appl., vol. 2019, no. 6, pp. 95-114, 2020.

[24] M. Růžička, Electrorheological fluids: modeling and mathematical theory, Lecture Notes in Mathematics, vol. 1748, Berlin: Springer-Verlag, 2000. 
[25] L.-L. Wang, Y.-H. Fan, W.-G. Ge, "Existence and multiplicity of solutions for a Neumann problem involving the $p(x)$-Laplace operator", Nonlinear Anal., vol. 71, no. 9, pp. 4259-4270, 2009.

[26] J. Yao, "Solutions for Neumann boundary value problems involving $p(x)$-Laplace operator", Nonlinear Anal., vol. 68, no. 5, pp. 1271-1283, 2008.

[27] C. Zhang, S. Zhou, "Renormalized and entropy solutions for nonlinear parabolic equations with variable exponents and $L^{1}$ data", Journal of Differential Equations, vol. 248, no. 6, pp. $1376-1400,2010$.

[28] D. Zhao, W. J. Qiang, X. L. Fan, "On generalized Orlicz spaces $L^{p(x)}(\Omega)$ ", J. Gansu Sci., vol. 9, no. 2, pp. 1-7, 1997 [in Chinese].

[29] A. Zimmermann, "Renormalized solutions for a nonlinear parabolic equation with variable exponents and $L^{1}$ data", Ph. D. Thesis, T. U. Berlin, 2010. 